\title{
Formation of the charged exciton complexes in self-assembled InAs single quantum dots
}

\author{
E. S. Moskalenko \\ A.F. Ioffe Physical-Technical Institute, RAS, 194021, Polytechnicheskaya 26, St. Petersburg, Russia and \\ Department of Physics and Measurement Technology, Linköping University, S-581 83 Linköping, Sweden \\ K. F. Karlsson, P. O. Holtz, ${ }^{\text {a) }}$ and B. Monemar \\ Department of Physics and Measurement Technology, Linköping University, S-581 83 Linköping, Sweden \\ W. V. Schoenfeld, J. M. Garcia, and P. M. Petroff \\ Department of Materials, University of California, Santa Barbara, California 93106
}

(Received 11 June 2002; accepted 30 August 2002)

\begin{abstract}
We have studied the low-temperature photoluminescence (PL) of the self-assembled InAs single quantum dots (QDs) using conventional micro-PL setup to detect PL from an individual QD. It is demonstrated, that at certain experimental conditions, what concerns the laser excitation energy, the laser power and the crystal temperature, several additional lines, redshifted relative to the ground state transition, appear in the PL spectra. These are interpreted in terms of charged exciton complexes which form due to the population of quantum dots with a nonequal amount of electrons and holes. The latter phenomenon is determined by the excess energies of photogenerated carriers and is proposed as an effective optical method to create and study charged exciton complexes in QDs. (C) 2002 American Institute of Physics. [DOI: 10.1063/1.1516871]
\end{abstract}

\section{INTRODUCTION}

Self-assembled quantum dots (QDs) can be considered as artificial atoms because QDs, in contrast to the structures of higher dimensionalities, effectively confine electrons $(e)$ and holes $(h)$ on the nanometer length scale in all three directions. This results in zero dimensional density of states as well as in the increased role of Coulomb interactions in QDs. ${ }^{1}$ The latter together with the very restricted number (typically 2) of particles which could be accommodated at the ground state of the QD, makes the role of multiparticle effects of great importance both from the point of view of fundamental research and for a variety of applications. Indeed, multiparticle states, consisting of a large number of $e$ and $h$ (equal or nonequal) in QDs, which have been studied theoretically ${ }^{1-5}$ and experimentally, ${ }^{6-13}$ determine the performance of a number of optoelectronic (electronic) devices, for example QD lasers and single-electron transistors, which in turn are considerably affected by the charge stored in the QD. ${ }^{14}$

This highlights the important role of multiparticle complexes with a nonequal number of $e$ and $h$, and hence, stimulates the study of charged exciton complexes, which in the simplest case consist of one exciton and one additional charge carrier (i.e., $e$ or $h$ ). Also more complex charged excitons with several charge carriers of the same sign can be formed. ${ }^{2}$ For example, strong dependence of the QD emission spectra on the number of extra electrons (up to 20) was predicted in Ref. 2, where the role of the band gap renormalization as well as the shifts of the spectral lines in the charged exciton complexes due to the exchange energy were

a)Electronic mail: poh@ifm.liu.se discussed in detail. Experimental evidence for the formation of few electron states in QDs was revealed in capacitance experiments ${ }^{7,11}$ and studies of interband transmission. ${ }^{9,10}$

The formation of negatively charged exciton complexes was demonstrated in photoluminescence (PL) experiments. ${ }^{6,8}$ However, optical studies ${ }^{8-10}$ were dealing with a large ensemble of dots, so the results inevitably include the effect of inhomogeneous broadening which prevents accurate measurements and, hence, detailed analysis of optical properties of the QD. That is why the studies of charged exciton phenomena in the luminescence of single dots are highly desirable.

Up to date, two approaches have been used to study charged exciton complexes in single QDs. One method is to use specially designed samples ${ }^{12}$ containing an $n$-doped layer with contacts. The number of electrons in the QD can then be tuned by an applied external voltage. This gives the possibility to control the average number of excess electrons in the dot. However, the applied voltage causes a shift of the entire spectrum and deforms the wave functions, which eventually leads to change in the interaction energies. The other method is to use samples where the QDs are initially filled with a random number of electrons from the background doping. ${ }^{13}$ The main drawback of this method is that the initial number of excess electrons depends on the arbitrary number of impurity atoms in the close vicinity of the investigated QD and hence hardly controllable.

In the present contribution we propose another method to create and study charged exciton complexes in PL from a single InAs/GaAs QD. This method is based on the possibility to collect carriers in the QD, photoexcited in the barrier (GaAs in our samples), from a rather large area (typically a few micrometers ${ }^{15}$ relative to the lateral size of a QD. The 
process of carrier diffusion depends not only on the carrier type $(e$ or $h)$, i.e., on the effective mass of the diffusing particles, but is essentially governed by the energy of the created carriers relative to the band gap of the excited material. The significant increase of the effective diffusion constant of photogenerated carriers in GaAs quantum wells with increasing both pump-photon energy and crystal temperature was experimentally demonstrated in Refs. 15 and 16, respectively. Thus, it is reasonable to expect that the significantly different diffusivities of $e$ and $h$ will lead to a population of QDs with a nonequal number of $e$ and $h$. This effect will be manifested by the appearance of charged exciton complexes in the PL spectra of individual QDs.

We here report on PL from a single QD of InAs taking advantage of a very low density (less than $10^{7} \mathrm{~cm}^{-2}$ ) of QDs measured by a conventional micro-PL ( $\mu$-PL) setup. Our results demonstrate that changes in the excitation energy and crystal temperature initiate the appearance in the $\mu$-PL spectrum of two emission lines down shifted in energy with respect to the ground state exciton PL line. Moreover, the PL intensity of these lines as well as the intensity of the ground state exciton shows a periodic behavior with the excitation energy (four periods are detected altogether). This observation together with the predicted energy shifts, based on a simple perturbation theory model, ${ }^{4}$ allows us to identify the two additional low-energy lines as being due to the exciton charged with one and two additional electrons, respectively.

\section{SAMPLES AND EXPERIMENTAL SETUP}

The samples studied were grown by molecular beam epitaxy in the Stranski-Krastanow mode on a semi-insulating GaAs (100) substrate (the growth procedure used is described in detail elsewhere). ${ }^{17}$ On the top of a $100 \mathrm{~nm}$ wide $\mathrm{GaAs}$ barrier layer, the QDs are formed from a $1.7 \mathrm{InAs}$ monolayers deposition. With a special growth technique ${ }^{17}$ to reduce the dot size and improve the size distribution, the QDs were covered with another $100 \mathrm{~nm}$ wide GaAs layer for protection. Transmission electron microscopy studies of analogously grown samples revealed that uncapped original dots are lens (hemispherical) shaped with a typical lateral size of $35 \mathrm{~nm}$ and a height of $4.5 \mathrm{~nm} .{ }^{17}$ Such a reduced height of the QDs as well as significant interdiffusion of GaAs during the growth procedure blueshifts the QDs PL to the spectral region of $1.34 \mathrm{eV} .{ }^{17}$ A certain sample region of very low dot density was chosen for our study, where the interdot spacing was estimated to be about $10 \mu \mathrm{m}$, well exceeding the resolving power of the $\mu$-PL setup used.

The QDs were studied by means of a conventional diffraction-limited micro-PL setup. To excite the QDs, a cw beam of a Ti-Sp laser tunable between 700 and $900 \mathrm{~nm}$ was used, which could be focused on the sample surface down to a $2 \mu \mathrm{m}$ spot in diameter by a microscope objective through a thin optical window of the continuous flow cryostat. The luminescence signal was collected by the same objective and dispersed by a single-grating $0.45 \mathrm{~m}$ monochromator combined with a $\mathrm{LN}_{2}$ cooled Si-charge coupled device camera, which gives the spectral resolution in the region of the stud-

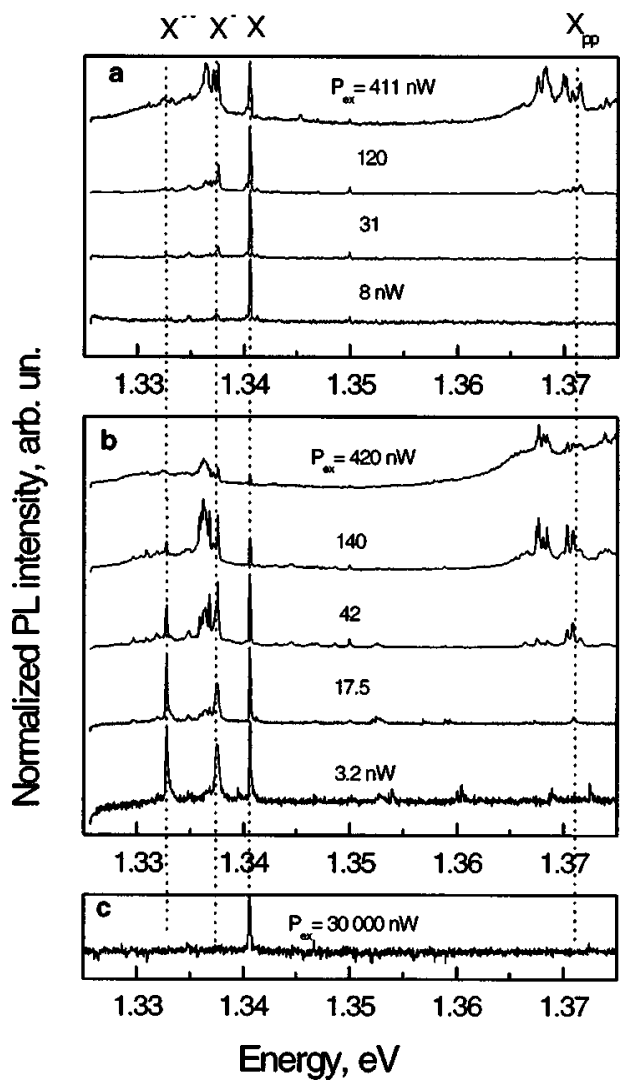

FIG. 1. Normalized PL spectra of an individual QD taken at $T=4 \mathrm{~K}$, for a number of $P_{\mathrm{ex}}$ at different pump-photon energies: (a) $h \nu=1.648 \mathrm{eV}$, (b) $h \nu=1.536 \mathrm{eV}$, and (c) $h \nu=1.433 \mathrm{eV}$.

ied PL of $0.15 \mathrm{meV}$. The crystal temperature $T$ was varied in the range $3.8 \mathrm{~K}<T<40 \mathrm{~K}$.

A magnified image of the sample surface was projected on a video camera, allowing a precise control of the laser position and focusing. Since any change in the temperature causes a sample drift, specially fabricated grids on the sample were used as a reference to locate the desired dot, as well as to effectively correct the position of the sample if it was moved due to the thermal drift.

Four single quantum dots located at different spatial positions of the sample were examined in this study. All of them revealed an analogous behavior although their dimensions were slightly different, as reflected by a small shift in the spectral positions of the PL lines. For consistency, we present the results taken from only one single quantum dot to demonstrate a typical behavior of QDs in the present study.

\section{EXPERIMENTAL RESULTS AND DISCUSSION}

Figure 1 shows PL spectra of an individual QD taken at a temperature of $T=4 \mathrm{~K}$ and different exciting photon energies $h \nu$ in a wide range of excitation powers $P_{\mathrm{ex}}$. At low $P_{\mathrm{ex}}$ in spectra taken with an excitation energy of $h \nu=1.648 \mathrm{eV}$ [Fig. 1(a)], a single line is revealed with a peak at $1.3406 \mathrm{eV}$ and labeled as $X$. This line is very narrow with its full width at half maximum of $0.15 \mathrm{meV}$, which is limited by our spectral resolution. The absence of other lines implies that the PL signal registered originates from the emission of a single QD. When increasing $P_{\text {ex }}$ up to $120 \mathrm{nW}$, two groups of lines 


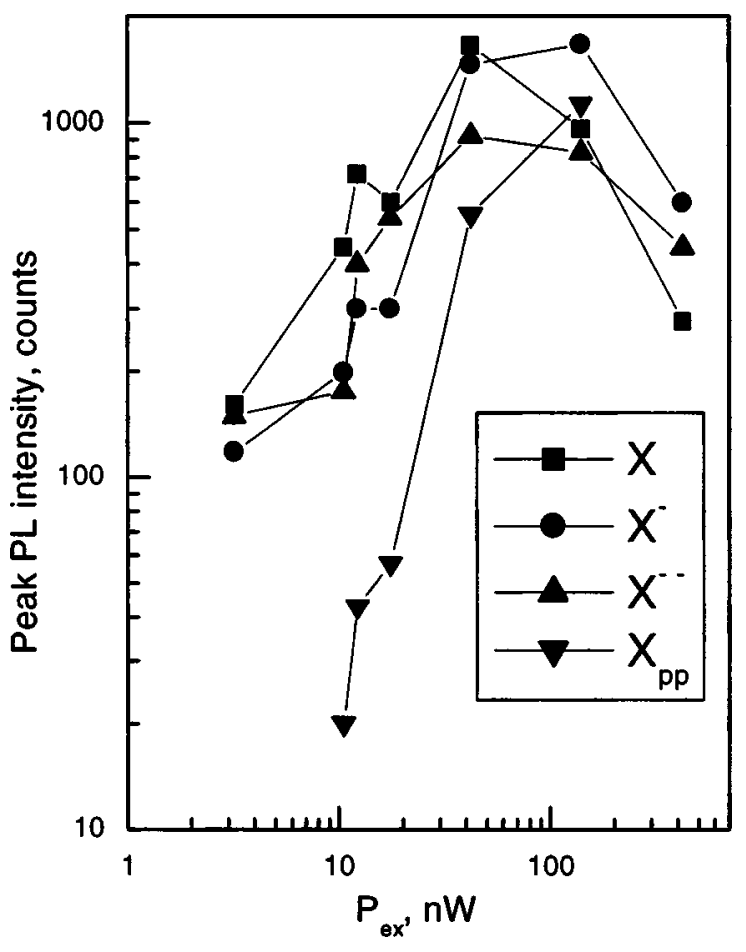

FIG. 2. Peak PL intensities of $X, X^{-}, X^{--}$, and $X_{p p}$ lines as a function of $P_{\text {ex }}$, taken at $h \nu=1.536 \mathrm{eV}$ and $T=4 \mathrm{~K}$.

appear in the spectrum. One group is down shifted in energy by approximately $3 \mathrm{meV}$ relative to the $X$ line, and the other group denoted as $X_{\mathrm{pp}}$ is blueshifted by $\sim 30 \mathrm{meV}$. Bayer et $a l .^{6}$ also observed the appearance of a similar group of lines, redshifted by 3-4 meV with respect to the $X$ line, which they interpreted in terms of biexcitonic emissions. At further increase of $P_{\mathrm{ex}}$, another group of lines $X_{\mathrm{pp}}$ grows in intensity faster and these lines dominate the PL spectra for $P_{\mathrm{ex}}$ $>500 \mathrm{nW}$.

At another exciting photon energy $h \nu=1.536 \mathrm{eV}$ [Fig. 1(b)], the PL spectra exhibit an analogous behavior at high $P_{\text {ex }}$, while for low $P_{\text {ex }}<40 \mathrm{nW}$, dramatic differences are detected. Indeed, the spectrum taken at $P_{\mathrm{ex}}=3.2 \mathrm{nW}[$ Fig. 1(b)] clearly shows the appearance of two lines (in addition to line $X$ ) peaking at 1.3375 and $1.3328 \mathrm{eV}$ and marked as $X^{-}$and $X^{--}$respectively. This is in sharp contrast to the results exhibited in Fig. 1(a), obtained at another excitation energy and low $P_{\text {ex }}$. Accordingly, it is possible to detect a various number of PL lines, dependent on the energy of the exciting photon, even at lowest excitation conditions.

All the low energy lines $\left(X, X^{-}\right.$, and $\left.X^{--}\right)$grow linearly with $P_{\mathrm{ex}}$, then saturate and even decrease their amplitudes as shown in Fig. 2, while the line $X_{\mathrm{pp}}$ (marked with a corresponding vertical dotted line in Fig. 1) reveals a quadratic dependence on $P_{\text {ex }}$ (Fig. 2). These characteristic excitation power dependencies together with the calculations of the characteristic energy separations between the ground and excited states in lens-shaped QDs of similar composition and sizes $^{5}$ allow us to ascribe the lines $X_{\mathrm{pp}}$ as the emission from the first excited state (both $e$ and $h$ occupy the corresponding $p$ level) of the QD while the low energy lines (around 1.34
$\mathrm{eV}$ ) originate from transitions related to the $e$ and $h$ from the lowest $s$ levels.

In the present study we will concentrate only on the lowest $P_{\text {ex }}$ limit, at which the most dramatic differences in PL spectra are revealed when changing $h \nu$.

To understand the dramatic changes in PL initiated by the tuning of the $h \nu$, we note first, that the difference occurs at lowest $P_{\mathrm{ex}}$ levels, so this fact together with the linear excitation power dependence of the $X, X^{-}$, and $X^{--}$lines allow us to exclude the possible influence of multiexcitonic complexes related to the occupation of QDs with more than one exciton ( $e-h$ pair) as an explanation. Second, the appearance of the $X^{-}$and $X^{--}$lines cannot be explained as an impurity related transition, i.e., an exciton complex consisting of an optically created exciton together with a carrier (positive or negative), which is trapped by the QD from the impurity atom located in the close vicinity of the QD. In that case, the QD could be populated with an exciton irrespective of the excitation conditions, and hence, the lines $X^{-}$and $X^{--}$would appear at any value of $h \nu$. Third, both excitation energies used, $h \nu=1.536 \mathrm{eV}$ and $h \nu=1.648 \mathrm{eV}$, are above the GaAs barrier and, hence, the observed changes in PL spectra cannot be understood in terms of the different influence of the below and above barrier excitations on the QD luminescence as was reported earlier. ${ }^{13}$

The possible difference for the two $h \nu$ 's used in case of Figs. 1(a) and 1(b) could be the different excess energies (with respect to the band gap $E_{g}$ ) of electrons, $\Delta E_{e}$, and holes, $\Delta E_{h}$, photoexcited in the GaAs barrier. A carrier can release its excess energy via the emission of optical or acoustic phonons. If $\Delta E_{e}\left(\Delta E_{h}\right)$ is larger than the optical phonon energy (for example longitudional optical phonon $\hbar \omega_{\mathrm{LO}}$ $=36 \mathrm{meV}$ in $\mathrm{GaAs})^{15}$ then the particle will effectively release its energy via the cascade emission of LO phonons. These processes are very fast (of the order of a few picoseconds) $)^{15}$ and, hence, the "cooling" time for the particle, for example $e$, will be determined by the residual excess energy $\Delta E_{e}^{r}=\Delta E_{e}-n \times \hbar \omega_{\mathrm{LO}}$, where $n=0,1,2, \ldots$ The latter can be released only via the emission of acoustic phonons which takes a much longer time. Hence, the higher the $\Delta E_{e}^{r}\left(\Delta E_{h}^{r}\right)$ is, the longer time is required for $e(h)$ to cool down, to form the exciton and to recombine. This eventually leads to the conclusion that particles with larger $\Delta E^{r}$ are able to move a longer distance before the exciton is formed, or in other words, they possess higher effective diffusivities. This corresponds to the experimental observation of increased effective diffusivities of carriers in GaAs quantum wells with increasing pump-photon energy. ${ }^{15}$ Also an oscillating behavior of the photoconductivity signal, which in turn reflects the carrier mobility and, hence, the diffusivity, has been measured in bulk GaAs, ${ }^{18}$ where up to 15 periods were detected as the excitation energy was increased.

Experimental evidence of the importance of diffusion processes in samples studied could be obtained by comparing the intensities of the wetting layer (WL) and the GaAs emission lines peaking at 1.445 and $1.515 \mathrm{eV}$, respectively, as shown in Fig. 3(a). The PL intensity of the WL line is greater by a factor of 250 than that of the GaAs although the 


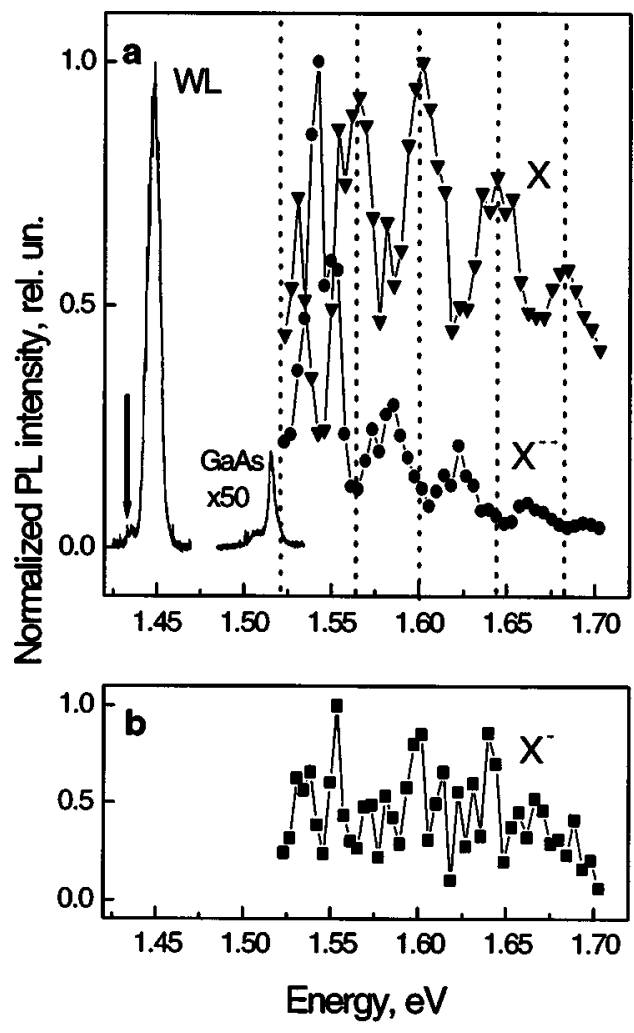

FIG. 3. The normalized peak PL intensities of (a) $X$ and $X^{--}$lines and (b) $X^{-}$line as a function of $h \nu$, taken at $T=4 \mathrm{~K}$ and $P_{\mathrm{ex}}=10 \mathrm{nW}$. The normalized PL spectrum of the WL and of the GaAs barrier is also shown for the same values of $T$ and $P_{\mathrm{ex}}$ at $h \nu=1.536 \mathrm{eV}$ (a).

absorption thickness of the WL is just $0.5 \%$ compared to the GaAs.

The difference in excess energies produced by the different $h \nu$ 's as well as the large difference in effective masses of $e$ and $h$ would imply that the numbers of $e$ and $h$ in the space region of the QD should be nonequal due to the essentially different diffusivities of the photoexcited carriers. Then it is reasonable to explain the three lines observed at low $P_{\mathrm{ex}}$ in Fig. 1(b) in terms of luminescence originating from different possible exciton configurations including the "pure" exciton transition $(e-h)$ and other configurations including excitons, which have captured extra particles ( $e$ or $h$, one or several, i.e., charged excitons). One of these three observed lines [Fig. 1(b)] corresponds to the single exciton $(e-h)$. In order to find out which line corresponds to the latter transition, the QD was excited with $h \nu=1.433 \mathrm{eV}$ which corresponds to excitation resonant with the localized states of the WL. This excitation energy is marked by a thick vertical arrow in Fig. 3(a) and the corresponding PL spectra of the QD is shown in Fig. 1(c). In order to monitor it, an extremely high $P_{\text {ex }}=30000 \mathrm{nW}$ was needed due to the very low absorption in the tail of the WL emission as compared to the bulk absorption in case of the excitation above the GaAs band gap. At these excitation conditions, no diffusion takes place and, hence, the QD is expected to be occupied with an equal number of $e$ and $h$. Indeed, we detect just a single line [Fig. 1(c)] which we ascribe to be the single exciton (e $-h$ ), marked as $X$ in Fig. 1. Consequently, the remaining two lines, marked as $X^{-}$and $X^{--}$are originating from con- figurations when the exciton $(e-h)$ has captured extra charge(s).

This fact strongly supports the assignment of the $X^{-}$and $X^{--}$lines done earlier, and implies that at an excitation with $h \nu=1.536 \mathrm{eV}$ [Fig. 1(b)], the QD is populated with additional electrons, while in the case of pumping with $h \nu$ $=1.648 \mathrm{eV}$ [Fig. 1(a)] there are no extra electrons captured to the dot. We attribute these differences to the different $\Delta E_{e}$ at these two excitations. To further elucidate the role of $\Delta E_{e}$, the dependence of the PL spectra on the $h \nu$ was investigated. Figure 3(a) shows the evolution of the PL peak amplitude of $X$ and $X^{--}$lines as a function of $h \nu$. The oscillating behavior of both $X$ and $X^{--}$is clearly seen. It is important to note that the amplitudes of $X$ and $X^{--}$behave in a reversed way, i.e., when $X$ gains its maximum value, $X^{--}$acquires its minimum amplitude and vice versa. This behavior is in full agreement with our model. Indeed, every time the QD is populated with extra electrons, the creation probability (and, hence, the PL amplitude) of the $X^{--}$line increases, while the corresponding probability for $X$ decreases.

This picture should repeat itself whenever the electron acquires the same $\Delta E_{e}^{r}$. The latter, in turn, takes place every time the pump-photon changes its energy by the value of $\Delta h \nu=\hbar \omega_{\mathrm{LO}} \times\left(1+m_{e}^{*} / m_{h}^{*}\right)$ which follows from the simple picture of band-to-band excitation in direct band gap semiconductors in the approximation of parabolic valence and conduction bands. Taking the electron and heavy hole effective masses in GaAs as $m_{e}^{*}=0.067 m_{0}$ and $m_{h}^{*}=0.45 m_{0}{ }^{15}$ and $\hbar \omega_{\mathrm{LO}}=36 \mathrm{meV}$, we obtain $\Delta h \nu=41.4 \mathrm{meV}$. Five vertical dotted lines in Fig. 3 are plotted with this energy separation starting from the value of $1.519 \mathrm{eV}$, which corresponds to the band gap $E_{g}$ of bulk GaAs. It is seen that they intersect characteristic minimum (maximum) amplitude values of the $X^{--}(X)$ lines. This circumstance, in turn, is consistent with our model, that it is the electrons, which initiate the dramatic changes observed in PL spectra when tuning the excitation photon energy.

It should be mentioned that the amplitude of the line $X^{-}$ [Fig. 3(b)] also shows an oscillating behavior with the excitation energy, although it is not so clear as in the case of the lines $X$ and $X^{--}$. This fact could be qualitatively understood on the basis of the following considerations: When $h \nu$ is changed, the probability to capture an extra electron to the QD is changed according to our model. If the capture probability increases, the amplitude of line $X^{-}$should increase, while correspondingly, the intensity of line $X$ will decrease. At the same time, some redistribution of the intensities of $X^{-}$ and $X^{--}$in favor of the line $X^{--}$should also take place, which gives rise to a decrease of the $X^{-}$line amplitude. Due to these competing processes, the analysis of the $X^{-}$line amplitude behavior with $h \nu$ is more complicated and less evident compared to the case of the line $X\left(X^{--}\right)$, shown in Fig. 3(a), which clearly decreases (increases) its amplitude in this particular case.

It is interesting to note that the excess energy of the heavy holes does not reach the value of $\hbar \omega_{\mathrm{LO}}$ in the excitation energy region under study, leading to a monotonous increase of the heavy-hole diffusivity. This could explain the 
decreasing amplitude of the intensity oscillations of the line $X^{--}$upon increasing the excitation energy $h \nu$ [Fig. 3(a)].

We will next discuss which type of carrier $(e$ or $h)$ an exciton can bind in case of lens (hemispherical) shaped QDs studied here. The problem of creation of charged exciton complexes, e.g., $(2 e-h)$ or $(e-2 h)$, was considered in Ref. 3 for both types of extra carriers and for two QDs spatial profiles; hemispherical and cone-shaped. The results of the detailed calculations show that in case of hemispherical shaped QDs, an electron is bound to the exciton, while the opposite situation takes place for the cone-shaped QDs. The exact calculations of the ground state energies ${ }^{2}$ predicted the existence of negatively charged excitons in lens-shaped QDs for the complexes consisting of one exciton and a number (up to 20) of additional electrons. By comparing the results of the detailed calculations with the results of a perturbation theory model, ${ }^{2}$ the conclusion was drawn that the exchange energy between the additional electrons and the "excitonic" electron gives the major contribution to the redshift of charged exciton complexes when the number of additional electrons increases.

On the basis of these theoretical predictions, ${ }^{2,3}$ we ascribe the lines marked as $X^{-}$and $X^{--}$to the charged exciton complexes with one and two additional electrons, respectively. Consequently, some characteristic energies, such as the binding energy of the single charged exciton $E_{\left(X^{-}\right)}^{b}$ and the exchange energy $E_{s p}^{(\mathrm{ex})}$ (between $s$ and $p$ electrons with parallel spins, as described later) can be deduced from the experiment: $\quad E_{\left(X^{-}\right)}^{b}=E_{(X)}-E_{\left(X^{-}\right)}=3.1 \mathrm{meV}$ and $E_{s p}^{(\mathrm{ex})}$ $=E_{\left(X^{-}\right)}-E_{\left(X^{--}\right)}=4.7 \mathrm{meV}$, where $E_{(X)}, E_{\left(X^{-}\right)}$, and $E_{\left(X^{--}\right)}$ correspond to the spectral positions of the $X, X^{-}$, and $X^{--}$ lines, respectively. To compare these experimentally obtained values with theoretical predictions we use the simple model developed in Ref. 4 to model the Coulomb blockade effect in capacitance measurements of InAs lens-shaped QDs. Lens-shaped QDs can be modeled with a parabolic confining potential ${ }^{5}$ which results in equidistant energy levels for $e$ and $h$ with energies of $\hbar \omega_{e}$ and $\hbar \omega_{h}$, respectively. In lens-shaped QDs of InAs, these energy quantities differ to a good approximation by a factor of 2 in favor of $\hbar \omega_{e} .{ }^{19}$

In the model, the formation process of the single charged exciton can be understood as follows. When an extra electron is added to the $\mathrm{QD}$, which ground state $(s s)$ is already occupied with a single exciton, two additional interactions will appear, namely an attractive interaction with the hole $E_{s s}^{\text {attr }}$ and a repulsive interaction between the two electrons $E_{s s}^{\text {rep }}$. The competition between these two terms will determine whether the extra electron will be bound or not. In the case of $X^{--}$, a third electron is added to the QD which is already occupied with two $s$ electrons and one $s$ hole. This electron could be added only to the $p$ state in order to minimize the total energy. If this $p$ electron has its spin parallel to that of the excitonic electron, the $X^{--}$line will be redshifted relative to the $X^{-}$line by the exchange interaction energy $E_{s p}^{(\mathrm{ex})}$. Following Ref. 4, the energies of interest can be written in the form

$$
E_{s s}^{\mathrm{rep}}=e^{2} /\left[4(2 \pi)^{1 / 2} \varepsilon_{0} \varepsilon_{r} l_{e}\right],
$$

$$
E_{s s}^{\mathrm{attr}}=e^{2} /\left[4(\pi)^{1 / 2} \varepsilon_{0} \varepsilon_{r}\left(l_{e}^{2}+l_{h}^{2}\right)^{1 / 2}\right] ; E_{s p}^{(\mathrm{ex})}=E_{s s}^{\mathrm{rep}} / 4,
$$

where $e$ is the elementary charge, $\varepsilon_{0}$ stands for the permittivity of vacuum, $\varepsilon_{r}=12.6$ is the dielectric constant, ${ }^{4}$ and $l_{e}\left(l_{h}\right)$ stands for the electron (hole) effective length

$$
l_{e}=\left(\hbar / m_{e}^{*} \omega_{e}\right)^{1 / 2}, l_{h}=\left(\hbar / m_{h}^{*} \omega_{h}\right)^{1 / 2} .
$$

To calculate the characteristic energies we take the values of the electron and hole effective masses to be $m_{e}^{*}$ $=0.07 m_{0}$ and $m_{h}^{*}=0.25 m_{0}\left(m_{0}\right.$ is the free electron mass), as experimentally evaluated ${ }^{4}$ for similar InAs/GaAs quantum dots used in the present study. We estimate $\hbar \omega_{e}$ and $\hbar \omega_{h}$ to be $\sim 20$ and $\sim 10 \mathrm{meV}$, respectively, using the experimentally obtained energy difference of $\sim 30 \mathrm{meV}$ between the $p p$ and $s s$ transitions [Figs. 1(a) and 1(b)]. By combining Eqs. (1a), (1b), and (2) we get: $E_{\left(X^{-}\right)}^{b}=E_{s s}^{\text {attr }}-E_{s s}^{\text {rep }}=2.6 \mathrm{meV}$ and $E_{s p}^{(\mathrm{ex})}=4.9 \mathrm{meV}$, which are in good agreement with the values of 3.1 and $4.7 \mathrm{meV}$ as derived from the PL spectra. For a comparison, we studied another QD with a different size, for which $E_{p p}-E_{s s}=45 \mathrm{meV}$, revealing energy differences $E_{\left(X^{-}\right)}^{b}=3.5 \mathrm{meV}$ and $E_{s p}^{(\mathrm{ex})}=6.2 \mathrm{meV}$, while according to the earlier mentioned calculation procedure corresponding values of 3.0 and $5.7 \mathrm{meV}$ were predicted.

It is worth mentioning that the excitation power evolution which is demonstrated for the two sets of spectra in Figs. 1(a) and 1(b) is totally consistent with the proposed model. Indeed, in the case when the QD is supposed to capture extra electrons [Fig. 1(b)] it is reasonable to expect the full occupation of the ground $(s)$ state of the QD at lower $P_{\text {ex }}$, than in the case of QD occupation with equal number of $e$ and $h$ [Fig. 1(a)]. As a result, one would expect the appearance of excited state transitions ( $X_{p p}$ lines) at lower $P_{\text {ex }}$ in case of Fig. 1(b) compared to the case of Fig. 1(a). This behavior is clearly demonstrated in Fig. 1 which shows, for example for $P_{\text {ex }} \sim 100 \mathrm{nW}$, the equal amplitudes for $s$ and $p$ states [Fig. 1(b)], while in the case of Fig. 1(a), these differ by a factor of 10 in favor of the ground state lines.

Another way to increase the effective diffusivity of the carriers is to increase the crystal temperature $T .{ }^{16}$ Since the electron mass is about seven times lighter than the heavyhole mass in GaAs, the electrons gain a higher diffusivity than the heavy holes, when the temperature is increased. The PL spectra shown in Fig. 4 demonstrate the temperature dependence at the three characteristic excitation energies $h \nu$. Due to the small thermal shift of the GaAs band gap, the excitation energy $h \nu$ was shifted by the same amount to keep the excess energy of photogenerated carriers, imposed by the photoexcitation, fixed. However, in the temperature region under study $(4-40 \mathrm{~K})$, this shift is small $(\approx 1 \mathrm{meV})$ and should not essentially affect the spectrum-even if not compensated. An analogous shift is also observable for the QD luminescence, as can be seen in Fig. 4. In Fig. 4(a), the excitation energy $h \nu$ is chosen in such a way, that solely the pure exciton line $X$ is created at $4 \mathrm{~K}$. It is clear that when the temperature is increased, there is a successive transfer of the intensity from the $X$ line to the redshifted lines $X^{-}$and $X^{--}$, indicating an increased probability for the QD to become charged. At $40 \mathrm{~K}$ the three lines have comparable intensities. Further, in Fig. 4(b) the excitation energy $h \nu$ is tuned (still 


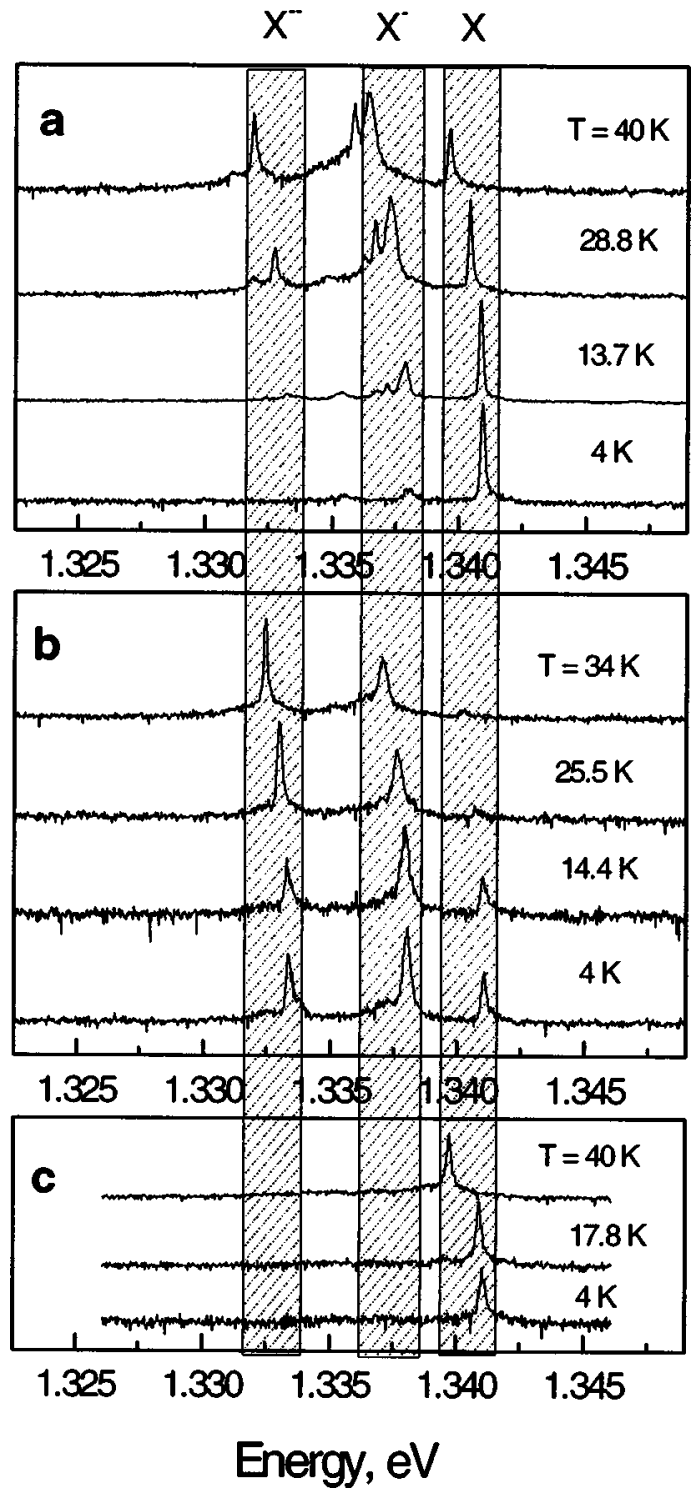

FIG. 4. Normalized PL spectra of a selected QD taken for a number of $T$ at different excitation energies: (a) $h \nu=1.686 \mathrm{eV}$ and $P_{\mathrm{ex}}=5 \mathrm{nW}$, (b) $h \nu$ $=1.557 \mathrm{eV}$ and $P_{\mathrm{ex}}=5 \mathrm{nW}$, and (c) $h \nu=1.433 \mathrm{eV}$ and $P_{\mathrm{ex}}=30000 \mathrm{nW}$.

remaining greater than $E_{g}$ ) to give comparable intensities for the three peaks at $4 \mathrm{~K}$. At $35 \mathrm{~K}$, the spectrum is already dominated by the line $X^{--}$, while the line $X$ has completely vanished. Extra attention should be paid on the relative intensities of the lines $X$ and $X^{--}$, which at these conditions are most sensitive to a change of the average number of excess electrons trapped in the QD. The monotonous decrease (increase) of the amplitude of $X\left(X^{--}\right)$line shows that an increased temperature effectively fills the QD with extra electrons.

To finally confirm that the temperature effect is due to an increased diffusivity and not due to an activation of localized electrons (for example electrons localized at heterointerface potential fluctuations, or by impurities caused by unintentional background doping), ${ }^{13}$ the sample was excited with an excitation energy $h \nu$ below the WL emission [shown by an arrow in Fig. 3(a)], while the temperature was increased [Fig. 4(c)]. Since the carriers in this case are excited to localized states of the WL and, hence, are totally immobile at low $T$, no temperature-induced diffusion is expected (unless the activation energy of localized electrons is less or comparable to $4 \mathrm{meV}$, which corresponds to $T=40 \mathrm{~K}$ ) in neither the GaAs nor the WL. Accordingly no peak except the pure exciton line $X$ is expected in the spectrum to appear with increasing $T$. On the other hand, if the extra electrons, which effectively populate the $\mathrm{QD}$ with increasing $T$, were due to the temperature activation of electrons, the spectrum should reveal qualitatively the same temperature behavior as for the excitation energies $h \nu>E_{g}$ [Figs. 4(a) and 4(b)]. Obviously the spectra in Fig. 4(c) are temperature independent, except for a redshift, similar to those revealed in Figs. 4(a) and 4(b). This fact supports our explanation presented for the exciton lines redistribution at elevated temperatures in terms of an increased photogenerated carriers diffusivity.

\section{CONCLUSIONS}

The PL spectra from single InAs QDs have been investigated as a function of the excitation power, pump-photon energy, and crystal temperature. For a fixed excitation power, the PL spectra show dramatic changes as a function of excitation energy: For certain pump-photon energies, two additional excitonic lines appear which are redshifted relative to the ground state exciton transition. A qualitative model is proposed, which predicts the crucial role of excess energy of the photogenerated electrons and explains the oscillating behavior of the PL amplitudes observed in the experiment. The appearance of these additional lines (or redistribution of PL lines) with increasing crystal temperature confirms the predominant role of carrier diffusivity in the occupation of the QD with extra electrons. All experimental data obtained together with estimates made by means of a simple perturbation theory model ${ }^{4}$ allowed us to identify the three lines observed in PL at low $P_{\mathrm{ex}}$ : The $X^{-}$and $X^{--}$lines are interpreted as excitons charged with one and two additional electrons, respectively. The pump-photon energy/crystal temperature influence on PL spectra is proposed as an interesting method to create charged exciton complexes in quantum dots in a controlled way.

\section{ACKNOWLEDGMENTS}

One of the authors (E.S.M.) gratefully acknowledges financial support of Svenska Institutet within the Visby Program and partial support of the Russian Academy of Sciences (Low-Dimensional Nanostructures, 2001).

${ }^{1}$ G. Medeiros-Ribeiro, F. G. Pikus, P. M. Petroff, and A. L. Efros, Phys. Rev. B 55, 1568 (1997).

${ }^{2}$ A. Wojs and P. Hawrylak, Phys. Rev. B 55, 13066 (1997).

${ }^{3}$ Ph. Lelong and G. Bastard, Solid State Commun. 98, 819 (1996).

${ }^{4}$ R. J. Warburton et al., Phys. Rev. B 58, 16221 (1998).

${ }^{5}$ A. Wojs, P. Hawrylak, S. Faffard, and L. Jacak, Phys. Rev. B 54, 5604 (1996).

${ }^{6}$ M. Bayer, T. Gutbrod, A. Forchel, V. D. Kulakovskii, A. Gorbunov, M. Michel, R. Steffen, and K. H. Wang, Phys. Rev. B 58, 4740 (1998); M. Bayer, A. Kuther, F. Schafer, J. P. Reithmaier, and A. Forchel, ibid. 60, R8481 (1999).

${ }^{7}$ S. Tarucha, D. G. Austing, T. Honda, R. J. van der Hage, and L. P. Kouwenhoven, Phys. Rev. Lett. 77, 3613 (1996).

${ }^{8}$ K. H. Schmidt, G. Medeiros-Ribeiro, M. Oestreich, P. M. Petroff, and G. 
H. Dohler, Phys. Rev. B 54, 11346 (1996); K. H. Schmidt, G. MedeirosRibeiro, and P. M. Petroff, ibid. 58, 3597 (1998).

${ }^{9}$ H. Drexler, D. Leonard, W. Hansen, J. P. Kotthaus, and P. M. Petroff, Phys. Rev. Lett. 73, 2252 (1994).

${ }^{10}$ R. J. Warburton, C. S. Durr, K. Karrai, J. P. Kotthaus, G. MedeirosRibeiro, and P. M. Petroff, Phys. Rev. Lett. 79, 5282 (1997).

${ }^{11}$ B. T. Miller, W. Hansen, S. Manus, R. J. Luyken, A. Lorke, J. P. Kotthaus, and S. Huant, Phys. Rev. B 56, 6764 (1997).

${ }^{12}$ F. Findeis, M. Baier, A. Zrenner, M. Bichler, G. Abstreiter, U. Hohenester, and E. Molinari, Phys. Rev. B 63, 121309 (2001).

${ }^{13}$ A. Hartmann, Y. Ducommun, E. Kapon, U. Hohenester, and E. Molinari, Phys. Rev. Lett. 84, 5648 (2000); A. Hartmann, Y. Ducommun, M. Bachthold, and E. Kapon, Physica E (Amsterdam) 7, 461 (2000).

${ }^{14}$ D. Bimberg, M. Grundmann, and N. N. Ledentsov, Quantum Dot Heterostructures (Willey, London, 1999).

${ }^{15}$ J. P. Wolfe, H. W. Yoon, D. R. Wake, and H. Morkoc, Semicond. Sci. Technol. 7, B240 (1992); H. W. Yoon, D. R. Wake, and J. P. Wolfe,
Phys. Rev. B 54, 2763 (1996).

${ }^{16}$ H. W. Yoon, D. R. Wake, J. P. Wolfe, and H. Morkoc, Phys. Rev. B 46, 13461 (1992); H. Hillmer, A. Forchel, S. Hansmann, M. Morohashi, E. Lopez, H. P. Meier, and K. Ploog, ibid. 39, 10901 (1989).

${ }^{17}$ K. H. Schmidt, G. Medeiros-Ribeiro, J. M. Garcia, and P. M. Petroff, Appl. Phys. Lett. 70, 1727 (1997); J.M. Garcia, T. Mankad, P. O. Holtz, P. J. Wellman, and P. M. Petroff, Phys. Rev. B 72, 3172 (1998); J. M. Garsia, G. Medeiros-Ribeiro, K. Schmidt, T. Ngo, J. L. Feng, A. Lorke, J. Kotthaus, and P. M. Petroff, ibid. 71, 2014 (1997).

${ }^{18}$ R. W. Shaw, Phys. Rev. B 3, 3283 (1971).

${ }^{19}$ As follows from the solution of the simple two-dimensional harmonic oscillator model, the ratio $\hbar w_{h} / \hbar w_{e}$ depends only on the effective masses and the depths of the confining parabolic potentials. Therefore, $\hbar w_{h} / \hbar w_{e}$ is expected to be close to 1/2 for the QDs studied in the present article, as obtained both experimentally, see R. J. Warburton et al., Phys. Rev. B 58, 16221 (1998) and theoretically, see A. Wojs, P. Hawrylak, S. Faffard, and L. Jacak Phys. Rev. B 54, 5604 (1996) for similar QDs. 